Canadian

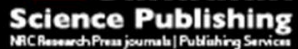

Canadian Journal of Physiology and Pharmacology Revue canadienne de physiologie et pharmacologie

\title{
Glutamine attenuates Obstructive Cholestasis in Rats via Farnesoid X Receptor -mediated Regulation of Bsep and Mrp2
}

\begin{tabular}{|r|l|}
\hline Journal: & Canadian Journal of Physiology and Pharmacology \\
\hline Manuscript ID & cjpp-2016-0389.R1 \\
\hline Manuscript Type: & Article \\
\hline Complete List of Authors: & $\begin{array}{l}\text { Liu, Bingli ; Department of General Surgery } \\
\text { Li, Yiming; Department of General Surgery } \\
\text { Ji, Hong; Department of General Surgery } \\
\text { Lu, Hongwei ; Department of General Surgery } \\
\text { Li , Hua ; Department of General Surgery } \\
\text { Shi, Yakun ; Department of Obstetrics and Gynecology }\end{array}$ \\
\hline Keyword: & Glutamine, Obstructive cholestasis, Bsep, Bile acid, FXR \\
\hline & \multicolumn{2}{|l}{} \\
\hline
\end{tabular}

SCHOLARONE ${ }^{\text {M }}$

Manuscripts 
1 Glutamine attenuates Obstructive Cholestasis in Rats via Farnesoid X Receptor

$2 \quad$-mediated Regulation of Bsep and Mrp2

3 Liu, Bingli ${ }^{1}$; Li, Yiming ${ }^{1, \uparrow} ;$ Ji, Hong $^{1}$; Lu, Hongwei ${ }^{1}$; Li , Hua ${ }^{1}$; Shi, Yakun ${ }^{2}$

$4 \quad{ }^{1}$ Department of General Surgery, The Second Affiliated Hospital of Xi'an Jiaotong University,

$5 \quad$ Xi'an 710004, China

$6 \quad{ }^{2}$ Department of Obstetrics and Gynecology, The Second Affiliated Hospital of Xi'an Jiaotong

7 University, Xi'an 710004, China

$8 \quad{ }^{\dagger}$ Correspondence to: Yiming Li, MD, PhD, Department of General Surgery, The Second

9 Affiliated Hospital of Xi'an Jiaotong University, 157 Xiwu Road, Xi'an 710004, China.

10 Email address: abgchy@stu.xjtu.edu.cn

11 Telephone: +86-029-87679246

12 Fax: +86-029-87679246

13

14

15

16

17

18

19

20

21

22 


\section{Abstract:}

24 To investigate the protective effect of glutamine (Gln) against obstructive cholestasis is in association with Farnesoid X receptor (FXR) activation. Obstructive cholestasis model was established in male SD rats by giving bile duct ligation (BDL). Serum biomarkers and H\&E staining were used to identify the amelioration of the hepatic injury degree of obstructive cholestasis rats after Gln treatment. Immunohistochemitry, RT-PCR, western blot, cultured primary rat hepatocytes with FXR knockdown and dual luciferase reporter assay were performed to elucidate the mechanisms underlying Gln hepatoprotection. We found that Gln treatment protected against obstructive cholestasis induced by BDL through alleviating serum biomarkers $(P<0.01$, respectively) and reducing hepotocyte injury. An up-regulation of hepatic efflux transporter (Shp, Bsep and Mrp2), an inhibition of hepatic uptake transporter (Ntcp) and bile acid synthesis enzyme Cyp7a1 expression were observed in BDL rats with Gln treatment in vivo. Furthermore, the regulatory effect of Gln on Bsep and Mrp2 expressions was abrogated after FXR knockdown in rat primary cultured hepatocytes. Luciferase assay HepG2 cells also illustrated FXR was a direct target for Gln treatment. In conclusion: The regulation of Bsep and Mrp2 expressions mediated by FXR might an important mechanism for Gln against obstructive cholestasis.

Keywords: Glutamine; Obstructive cholestasis; Bsep; Bile acid; FXR; Mrp2 


\section{Introduction}

Obstructive cholestasis is charactered by a dramatically increased bile acid levels in liver and serum, which eventually results in impairment of hepatic canalicular bile efflux, acute hepatic toxicity, bile duct proliferation, fibrosis, and even cirrhosis. Various factors can cause cholestasis such as drugs, viral hepatitis, or genetic disorders. Currently, drugs like ursodeoxycholic acid (UDCA) are referred as the primary medicines for the treatment of cholestatic disorders; however, the treatment efficacy is very limited and not satisfied in clinic. Thus, it is an urgency to develop a novel agent to effectively resist the cholestatic disorders.

The pathogenesis of cholestasis may result from the dysfunctions of hepatic transport systems, such as the impairment of bile formation or bile flow in the hepatocyte (Marin et al. 2015; Zollner and Trauner 2006). Bile acids (BAs) are synthesized by hepatocytes from cholesterol in the liver, mainly involved in regulating bile flow in the hepatocytes and cholangiocytes through stimulating some signaling pathways (Kullak-Ublick et al. 2000; Mazuy et al. 2015; Zollner et al. 2006).The intracellular ligand-activated nuclear receptor (NR), farnesoid X receptor (FXR, NR1H4) as well as various transporters and enzymes have been considered to play pivotal roles in regulating BAs homeostasis in the liver, of which FXR is the master regulator of hepatic BAs synthesis and secretion (Ding et al. 2015; Suchy and Ananthanarayanan 2006). BAs are firstly synthesized by the transcription of the rate-limiting enzyme cholesterol 7a-hydroxylase (CYP7a1) in hepatocytes (Trauner et al. 2005); Then in the liver, an orphan nuclear hormone receptor named small heterodimer partner (Shp; NR0B2) is transcriptionally upregulated by BAs- FXR interaction, which 
reversely represses Cyp7a1 activity (Zhou and Hylemon 2014); In addition, the activiation of FXR can negatively regulate the expression of $\mathrm{Na}^{+}$/taurocholate cotransporting polypeptide (Ntcp), resulting in inhibiting the uptake of BAs into the hepatocytes (Stieger 2011); while increase bile acid efflux by two ATP-binding cassette (ABC) transporters bile salt export pump (Bsep) and multidrug resistance-associated protein 2 (MRP2) to induce BAs excretion from the bile canalicular membrane into the canalicular lumen (Stieger and Beuers 2011).

Glutamine (Gln) as a conditionally essential amino acid is primarily formed and stored in lung and skeletal muscle. The nutritious effect of Gln has been illustrated in various tissues including fibroblasts, lymphocytes, small intestine enterocytes, and macrophages. Though Gln has no antioxidant effect, it can play a critical role against stressful stimuli, such as infection, inflammation, ischemia and even cancer (Miller 1999). In recent years, more scientific attention has been attracted to the protective effects of Gln against hepatic diastases. However, whether Gln against obstructive cholestasis is mediated by FXR and its down regulatory genes Mrp2 and Bsep still remains further investigation.

Thus, in this study, rat model of obstructive cholestasis was built to assess the hepatoprotective and choleretic effect of Gln, which may allow a better understanding of the molecular pathway regulated by Gln in cholestasis and provide potential pharmacological evidence targeting the involved regulatory networks.

\section{Materials and Methods}

\section{Obstructive cholestasis rat model build}

Adult male Sprague-Dawley rats weighing 250-300 g were purchased from the 

and tap water ad libitum. Obstructive cholestasis animal models were built using a modified method in previous study(Li and Chung 2001). Rats were subjected to laparotomy twice. All procedures were performed under anesthesia with $3 \%$ pentobarbital sodium $(1.0 \mathrm{ml} / \mathrm{kg})$.

Obstructive cholestasis was induced by double ligation of the common bile duct and Sham group (SH) was produced by separating bile duct locally but not ligated. To minimize tissue handling and dissection the duodenum was extracted with an ophthalmic muscle hook and the common bile duct isolated using very fine forceps. After bile duct ligation (BDL), all SD rats were randomly divided into 4 groups $(n=10)$ and treated as follows: Sham + normal saline $(\mathrm{SH}+\mathrm{NS})$ group, the rats in SH group were orally administrated with $0.9 \%$ normal saline at the dose of $10 \mathrm{mg} / \mathrm{kg}$ per day for 14 consecutive days; BDL + NS group, the rats with BDL were orally administrated with $0.9 \%$ normal saline at the dose of $10 \mathrm{mg} / \mathrm{kg}$ per day for 14 consecutive days; BDL+ Gln group, rats with BDL were given Gln (Sigma, MO, USA) at the dose of $200 \mathrm{mg} / \mathrm{kg}$ orally per day for 14 consecutive days; BDL+UDCA group, rats with BDL were given ursodeoxycholic acid (UDCA, Sigma) at dose of $50 \mathrm{mg} / \mathrm{kg}$ orally per day for 14 consecutive days. After final dose of injection, rats were fasted for the following $24 \mathrm{~h}$, the animals were then succumbed and serum and liver tissue samples were obtained immediately. This study was in accordance with the provisions of the Declaration of Helsinki and approved by Animal Research Ethics committee of Xi'an Jiaotong University. 
107

108

109

110

111

112

113

114

\title{
Histological and biochemical assessment
}

\author{
Liver tissues were fixed immediately in $10 \%$ neutral buffered formalin and
} paraffin-embedded blocks were made. Serial sections $5 \mu \mathrm{m}$ thick were stained with H\&E for evaluation of portal inflammation, hepatocellular necrosis and inflammatory cell infiltration. Sections were examined under a light microscope (Eclipse 50i, Nikon, Tokyo, Japan). Liver function markers of serum alanine aminotransferase (ALT), aspartate aminotransferase (AST), alkaline phosphatase (ALP), $\gamma$-glutmyltranspeptidase ( $\gamma$-GTP), total cholesterol (TC), direct bilirubin (DBIL), total bilirubin (TBIL) and biliary total bile acids (TBA) were analyzed using a commercially available clinical test kit with an automatic biochemical analyzer (AU2700, Olympus, Tokyo, Japan).

\section{Immunohistochemistry and morphometry}

The immunohistochemical staining was performed on sections of liver samples. The sections were incubated overnight at $4^{\circ} \mathrm{C}$ with the anti-Bsep antibody (rabbit polyclonal antibody against Bsep, bs-1954R, Boisynthesis Biotechnology, Beijing, China) at a dilution of 1:200, and the anti-Mrp2 antibody (mouse monoclonal antibody against Mrp2, SC-59611, Santa Cruz, CA, United States) at a dilution of 1:300, respectively. Following several rinses in phosphate buffered solution; the sections were incubated with the biotinylated goat anti-rabbit IgG antibody or biotinylated goat anti-mouse IgG antibody (Boisynthesis Biotechnology, Beijing, China) for $30 \mathrm{~min}$ at $37^{\circ} \mathrm{C}$. Finally, the sections were colored with $\mathrm{DAB}$ at room temperature for 1-15 min, counterstained with hematoxylin for $30 \mathrm{~s}$, dehydrated through gradient ethanol, cleared in xylene and then mounted with permount. Images were obtained 
128

131

144 145

using a light microscope (Eclipse 50i, Nikon, Tokyo, Japan). Immunoreactivity of Bsep and Mrp2 in rat liver was morphometrically identified by the Image Pro Plus 6.0 image analysis software system (Media Cybernetics, MD, United States).

\section{Quantitative real time (qRT-PCR) analysis}

Total RNA was extracted from $100 \mathrm{mg}$ snap-frozen liver tissue using RNAiso plus reagent (Takara, Dalian, China) according to the manufacturer's instructions. The quality and quantity of RNA were assessed using 1\% agarose gel electrophoresis and spectrophotometric analysis of 260/280 ratios. RNA was reversely transcribed with oligo primer using PrimeScript $^{\mathrm{TM}}$ RT reagent Kit (Takara, Dalian, China). The primers of genes were also designed by Takara, and no significant homologous sequence was discovered using NationalCenter for Biotechnology Information, The Basic Local Alignment Search Tool (NCBI BLAST). The housekeeping gene $\beta$-actin was used as an internal reference gene to normalize the transcript levels. The primer sequences of FXR, Bsep, Mrp2, Cyp7a1, Ntcp and Shp were listed in Table 1. qRT-PCR was performed on a Step one plus real-time PCR system (ABI, CA, United States) using the $\mathrm{SYBR}^{\circledR}$ Premix Ex Taq ${ }^{\mathrm{TM}}$ II reagent Kit (Takara, Dalian, China) under the following conditions: $30 \mathrm{~s}$ at $95^{\circ} \mathrm{C}$ followed by 40 cycles of $5 \mathrm{~s}$ at $95^{\circ} \mathrm{C}$ and $30 \mathrm{~s}$ at $60^{\circ} \mathrm{C}$. The relative expression of the target gene was represented by the $2^{-\Delta \Delta \mathrm{CT}}$ value of the tested sample.

\section{Western blot analysis}

The proteins were extracted using Protein Extraction Kit (Promega, WI, United States). Thirty micrograms of proteins were mixed with the sample buffer for sodium dodecyl sulfate 
149 polyacrylamide gel electrophoresis (SDS-PAGE). The electrophoresis was performed and the

150 proteins were transferred to the polyvinylidene fluoride (PVDF) membranes. The membranes

151 were incubated with the primary antibodies, including FXR (H-130), Bsep (F-6), Mrp2

152 (M2III-5), Ntcp (M-130), Shp (F-12) and CYP7a1 (H-58) (Santa Cruz, CA, US) at a dilution

153 of 1:200, respectively, while shaking at $4^{\circ} \mathrm{C}$ overnight. Then the PVDF membranes were

154 incubated with goat anti-rabbit IgG-HRP antibody or goat anti-mouse IgG-HRP antibody

155 (Boisynthesis Biotechnology, Beijing, China) $(1: 1,000)$ at room temperature for $2 \mathrm{~h}$ and were

156 colored by enhanced electrochemiluminescence reagents (32109, Pierce, IL, United States)

157 after the membrane had been washed repeatedly. Finally, the membranes were imaged and

158 analyzed by the SyngeneG: BOX analytical system and normalized using mouse monoclonal

159 anti- $\beta$-actin antibody (sc-47778, Santa Cruz, CA, US).

160 Isolation and culture of primary rat hepatocytes

161 Hepatocytes from male SD rats were isolated by using a modified two-step collagenase

162 digestion method as described previously (Shen et al. 2012). Hepatocytes were washed with

163 WME medium supplemented with $100 \mathrm{U}$ penicillin/streptomycin (Sigma, USA) and 10\%

164 heat-inactivated fetal bovine serum (FBS) (Gibco, USA). Then the hepatocytes were cultured

165 in plates pre-coated with rat tail collagen (Shengyou Biotechnology, Hangzhou, China) and

166 incubated for $12 \mathrm{~h}$ at $37^{\circ} \mathrm{C}$, then replaced with the fresh DMEM supplemented with $10 \% \mathrm{FBS}$,

$16720 \mathrm{mg} / \mathrm{L}$ hepatocyte growth-promoting factor (HGF) (Langdun Biotechnology, Shanghai,

168 China), $0.5 \mathrm{mg} / \mathrm{L}$ insulin (Sigma, MO, USA) and 0.1 $\mu$ M Dexamethasone (Sigma, MO, USA)

169 and incubated for another $12 \mathrm{~h}$ before treatment. The viability of hepatocytes was measured 
170 by trypan blue exclusion and only cells with viability more than $90 \%$ could be used for

171 further study.

172 FXR siRNA treatment in cultured primary rat hepatocytes

173 Cultured primary rat hepatocytes were seeded onto 24 -well plate at a density of $1 \times 10^{5}$

174 cells per well. $12 \mathrm{~h}$ later, the cells were treated with vehicle (0.1\% DMSO) or siRNA. For

175 RNA silencing experiment, siRNA specific targeting at mouse FXR sequence AGG TTC CTT

176 TCT ATG TTT ATA TC were designed as follows: sense: 5'- UAU AAA CAU AGA AAG

177 GAA CCU -3'; Anti-sense: 3'- GUU CCU UUC UAU GUU UAU AUC-5' (GenePharma,

178 Shanghai) and transiently transfected to cultured primary rat hepatocytes using Lipofectamine

1792000 (Invitrogen, US) and incubated for $24 \mathrm{~h}$. After that, $100 \mu \mathrm{M}$ Gln were added to the

180 medium for another $24 \mathrm{~h}$. the cells were harvested for western blot analysis.

\section{Dual luciferase reporter assay}

182 FXR expression plasmid was constructed by cloning the FXR gene sequence into

183 pCI-neo mammalian expression vector (Promega). Besp luciferase reporter vector was

184 constructed by cloning the upstream DNA fragment of promoter region into the luciferase

185 vector pGL4.14 [Lcu2/Hygro] (Promega) (Sang et al. 2008). For vitro transfection study,

186 HepG2 cells were seeded in 24-well plates at a density of $1 \times 10^{5}$ cells/ well in DMEM media

187 supplemented with $10 \%$ FBS and cultured for $24 \mathrm{~h}$. Then, the cells were cotransfected with

188 FXR expression plasmid, Bsep promoter luciferase reporter vector and the null-Renilla

189 luciferase plasmid as an internal control. After transfection for $24 \mathrm{~h}$, cells were treated with

190 vehicle (0.1\% DMSO), $100 \mu \mathrm{M}$ CDCA (Sigma, MO, USA) or 10, 40, 70, $100 \mu \mathrm{M}$ Gln for 24 
191 h. the luciferase activities were measured with a Dual-luciferase Reporter assay System

192 (Promega) according to manufactuer's instrunctions. The firefly luminescence was

193 normalized to the Renilla luminescence signal, and the ratio of treatment over control served

194 as fold activation.

195 Statistical analysis

196 All results were expressed as mean \pm standard deviation (SD). Statistical analyses

197 between two groups were performed by a Student's $t$-test and multiple comparisons were

198 performed by a one-way ANOVA. A $P<0.05$ was considered statistically significant.

\section{Results}

200 Morphological observation and liver function test of obstructive cholestasis rat

201 After BDL, rat skin stained yellow and lethargy; the obstructive rats became depressed,

202 inactive and anorectic gradually and passed dark yellow urine. During and after the biliary

203 drainage procedures, hemorrhage and dehydration were the main death reasons. Finally, 40

204 rats were enrolled into this study and treated as follows: the BDL rats were randomly injected

205 with Gln (BDL + Gln group), UDCA (BDL + UDCA group), or normal saline (BDL + NS

206 group) for 14 days. When compared to SH + NS group, the urine and skin color of BDL + NS

207 rats still stained yellow and showed serious depression with poor appetite, lighter weight, and

208 reduced activity. While in BDL + Gln and BDL + UDCA groups, the skin and urine of these

209 rats gradually returned to normal and their appetite and activity recovered remarkably.

\section{Levels of Serum Hepatic Biomarkers in Rats}

211 To study the liver biomarker changes in different treated rats, ALT, AST, ALP, $\gamma$-GTP, TC, 
212 DBIL, TBIL and TBA were analyzed. As shown in figure 1, the biochemical indicator levels

213 ALT, AST, ALP and $\gamma$-GTP in BDL + NS group were significantly increased when

214 compared to $\mathrm{SH}+\mathrm{NS}$ control group $(P<0.01$, respectively). Whereas, after treatment with

215 Gln or UDCA for 14 days in BDL rats, their levels gradually restored and showed a declined

216 tendency $(P<0.01$, respectively). Furthermore, consistent with serum biochemistry findings,

217 BDL-induced elevation of bile salts including TC, DBIL, TBA and TBIL were also reduced

218 by Gln and UDCA ( $P<0.05$, respectively). Notably, there was no significant difference

219 between Gln and UDCA treatments. Taken together, these results directly illustrated that Gln

220 possessed the function in attenuating BDL-induced obstructive cholestasis.

\section{Gln attenuated liver damage in obstructive cholestasis rat}

222 To further investigate the histological changes of obstructive cholestasis in rat tissues,

223 the liver sections stained with H\&E were determined by histopathological assessment.

224 Inflammatory cell infiltration, hepatocyte necrosis, bile duct proliferation, and liver fibrosis

225 were graded by (-, negative; + , slight; ++ , moderate; +++ , severe), and shown in Table 2 and

226 Figure 2. Histopathological study of sham operated rats revealed a normal liver lobular

227 architecture and no pathological changes in livers. Whereas, the liver of obstructive rats (BDL

$228+$ NS) showed severe bile duct proliferation and areas of confluent hepatocyte necrosis,

229 fibrous expansion of the portal tracts with severe fibrosis and even pseudolobule formed (Fig.

2302 and Table 2). Our results also found that after treatment with Gln and UDCA, the

231 histopathologic parameters in BDL + Gln and BDL + UDCA groups were markedly decreased,

232 and the liver displayed normal morphological features of hepatocytes and preserved lobular 
233 architecture with only mild bile duct proliferation and slight inflammatory cell infiltrationin

234 comparison to $\mathrm{SH}+\mathrm{NS}$ rats. Thus, consistent with the results of serum biomarkers detection,

235 the histological examination demonstrated that Gln supplementation alleviated BDL-induced

236 liver damage.

237 Bile efflux transporter Bsep and Mrp2 distributions in obstructive cholestasis

238 To elucidate the mechanism underlying the protective effect of Gln on obstructive

239 cholestasis, the canalicular efflux transporter Bsep and Mrp2 were determined by

240 immunohistochemical staining. The integrated optical density (IOD) was taken with the

241 Image-Pro Plus 6.0 software to qualify the stained density. As shown in Figure 3A, the

242 expression levels of Bsep and Mrp2 were mainly localized in the basolateral membrane of

243 hepatic cells. The expressions of Mrp2 and Bsep in the BDL + NS group were significantly

244 decreased in comparison to $\mathrm{SH}+\mathrm{NS}$ group $(P<0.01$, respectively) (Fig. 3). After treatment

245 with Gln and UDCA for 14 days in BDL group, the expressions of Bsep and Mrp2 were

246 significantly re-enhanced to the compatible level of SH group $(P<0.01$, respectively).

247 Alternation of bile acids regulatory molecules of obstructive cholestasis involved in Gln

248 treatment

249 To further verify the hepatoprotective effect of Gln on obstructive cholestasis was related

250 to the restoration of bile flow system. Western blot and RT-PCR were used to investigate the

251 alterations of hepatic key molecules including FXR, Bsep, Mrp2, Cypa1, Ntcp and Shp in

252 livers of rats with different treatments. The mRNA and protein levels of FXR, Bsep, Mrp2

253 and SHP were remarkably decreased, while Ntcp and Cyp7a1 expressions were significantly 
254 increased in BDL + NS group than in SH + NS group $(P<0.01$, respectively); In contrast, 255 after treatment with Gln and UCDA for 14 days, the expressions of FXR, Bsep, Mrp2, Shp, 256 Ntcp and Cyp7a1 were restored to the normal levels when compared to the SH + NS group 257 ( $P>0.05$, respectively) (Fig. 4). However, no significant difference was found between Gln 258 and UCDA treatments. Taken together, these data strengthened the hypothesis that Gln plays a 259 crucial role in protecting liver against obstructive cholestasis through bile acid synthesis and 260 bile efflux transporter pathway.

\section{The effect of GIn is abrogated by FXR silencing in rat primary cultured hepatocytes}

263 hepatic bile acid transport are downstream target genes of FXR. We hypothesized that Gln

264 may activate FXR to regulate expression level of genes in bile acid homeostasis. To verify 265 this hypothesis, siRNA was used to knock down the expression of FXR in cultured primary 266 rat hepatocytes. Western blot confirmed that the FXR expression was hardly detected after 267 specific siRNA targeting FXR transfection. In addition, the expressions of Mrp2 and Bsep 268 induced by Gln were remarkably abrogated by FXR silence $(P<0.01$, respectively) (Fig. 5A 269 and B). Therefore, it was further demonstrated the involvement of FXR activation in the 270 hepatoprotective effect of Gln against BDL-induced liver injury.

\section{Gln increased Bsep expression by serving as a partial FXR agonist}

272 Since significant increase expression of Bsep was detected in BDL rats with Gln 273 treatment, we then investigated whether this effect was directly caused by the main upstream 274 regulator FXR. Dual luciferase reporter assay was firstly performed in HepG2 cells 
275 cotransfected with FXR expression plasmid and FXR target gene Bsep promoter reporter

276 vector. As demonstrated in Figure 5C, CDCA $(100 \mu \mathrm{M})$, a characterized FXR agonist,

277 significantly increased the luciferase activity of FXR reporter gene compared to vehicle group

$278(P<0.01)$, while Gln (at doses of $10,40,70$ and $100 \mu \mathrm{M})$ also significantly increased the

279 luciferase activity of FXR at a dose-dependent manner $(P<0.05, P<0.05, P<0.01, P<0.01$,

280 respectively), which directly demonstrated the positive regulatory role of Gln on FXR

281 activation.

282 Discussion

283 Bile acids (BAs) as the major composition of bile, plays a pivotal role in lipometabolism

284 (Geier et al. 2007; Morgan et al. 2016). The impairment of bile secretion and flow could lead

285 to cholestasis syndrome such as lesions, primary sclerosing cholangitis, epatocellular and

286 cholangiocellular secretory defects (Matsuzaka et al. 2015). Furthermore, Tarantino et al.

287 have reported that acute drug-induced liver injury (DILI) in patients suffering from

288 non-alcoholic fatty liver disease (NAFLD) could present as a cholestatic form (Tarantino et al.

289 2007), because it's well known that NAFLD patients with biochemical cholestasis have a

290 histological picture of bile damage as Sorrentino et al. suggested (Sorrentino et al. 2005).

291 Previous researches have demonstrated that if the activation of FXR is blocked, liver

292 cholestasis could be aggravated by increasing the endogenous synthesis of BAs while

293 decreasing bile acid efflux ( $\mathrm{Li}$ and Guo 2015; Trauner et al. 1998). Current studies also

294 reported that knockout of the expression of Bsep could induce the occurrence of cholestasis in

295 rats (Funk et al. 2001). Moreover, the dysregulated expressions of Bsep and Mrp2 contributed 
to the onset of related cholestiatic diseases such as intrahepatic cholestasis of pregnancy (ICP) in adults and Dubin-Johnson syndrome (Cui et al. 2009; Kajihara et al. 1998; Wendum 2010). Donner et al. also declared that zonal downregulation of Bsep in obstructive cholestasis was associated with portal inflammation and is mediated by TNF-alpha and IL-1beta (Donner et al. 300 2007).

Aldemir et al. has reported that Gln could reduce the incidence of bacterial translocation and preserve intestinal mucosal integrity in common bile duct ligated rats (Aldemir et al. 2003). Xu et al. demonstrated that preconditioning with Gln significantly improved hepatic

304 structure and function by inhibiting reactive oxygen species, inflammation, and hepatocyte 305 apoptosis after ischemia/reperfusion injury in rats with obstructive jaundice (Xu et al. 2014).

306 Alecrim et al. indicated that Gln supplementation had no positive effect on colonic scar 307 strength in rats with extrahepatic biliary obstruction (Alecrim et al. 2015). Thus, it is urgent to 308 deeply reveal the hepatoprotective mechanism underlying Gln on obstructive cholestasis. In 309 the present study, Gln apparently mitigated serum transaminases in OJ-induced intrahepatic 310 cholestasis rats such as ALT, AST, ALP, $\gamma$-GTP and bile acids TC, TBIL, DBIL, TBA levels, 311 which were commonly used as crucial criteria for the diagnosis of hepatobiliary disorders 312 (Knight 2005; Lalisang 2012; Scheig 1996). Furthermore, H\&E experiment revealed that 313 obstructive cholestasis rats with Gln treatment remarkably alleviated severe necrotic and 314 degenerative changes of hepatobiliary cells, the bile duct proliferation, and the inflammatory 315 cell infiltration, indicating the hepatoprotective effects of Gln involved in obstructive 316 cholestasis. 
place at the basolateral membrane of hepatocytes and is mediated through Ntcp activation,

331 Gln as the key regulatory factor for BAs may become the effective therapeutic agent against obstructive cholestasis.

334 the significant effect of Gln on gene expression of Bsep and Mrp, which are the PXR target genes, in vitro experiments was performed in cultured primary rat hepatocytes, FXR siRNA 
338 Gln mediated activation of FXR pathway against BDL-induced cholestasis. Moreover, 339 previous study has demonstrated that Bsep was predominantly regulated by FXR (Song et al. 340 2013). In this study, we speculated that Bsep upregulation in BDL rats with Gln treatment 341 might be due to FXR agonism. The dual luciferase reporter assay confirmed that Gln could 342 stimulate FXR activation dose-dependently in HepG2 cells, which was similar to the effect of 343 FXR agonist CDCA. In the past decade, FXR has been regarded as a potential therapeutic 344 target in the treatment of cholestatic liver disorders. Therefore, we proposed that the role of 345 Gln in FXR agonism may also contribute to its anticholestatic effect.

346 In conclusion, we suggested that the involvement of Bsep and Mrp2 played crucial roles 347 in the process of Gln treatment in obstructive cholestasis, its effect might via activating FXR 348 pathway, and resulted in suppressing accumulation of TB, DB, and TBA in the liver through 349 increase in hepatic efflux, which might give new insight into this hepatic disease and obtain 350 effective therapeutic strategies. 


\section{Acknowledgement}

360 We would like to thank Dr. Yiming Li for his insightful comments and suggestions.

361

362 Conflict of interest and disclosure

363 The authors declare that no competing interest exists.

364

365

366

367

368

369

370

371

372

373

374

375

376

377

378

379 


\section{Reference}

Aldemir, M., Geyik, M.F., Kökoğlu, O.F., Büyükbayram, H., Hoşoğlu, S. and Yağmur, Y. 2003. Effects of ursodeoxycholic acid, glutamine and polyclonal immunoglobulins on bacterial translocation in common bile duct ligated rats. ANZ. J. Surg. 73 (9): 722-726. PMID:12956789.

Alecrim, H.M., Duarte, S.A., Amaral, M.E., Diogenis, F., Carneiro, F.P. and Sousa, J.B. 2015. Effect of glutamine supplementation on left colon healing in rats with extrahepatic biliary obstruction. Acta. Cir. Bras. 30 (1): 73-79. doi:10.1590/S0102-86502015001000010. PMID: 25627274.

Chen, H., Huang, X., Min, J., Li, W., Zhang, R., Zhao, W., et al. 2016. Geniposidic acid protected against ANIT-induced hepatotoxity and acute intrahepatic cholestasis, due to Fxr-mediated regulation of Bsep and Mrp2. J. Ethnopharmacol. 179 (1): 197-207. doi:10.1016/j.jep.2015.12.033. PMID:26723467.

Cui, T., Liu, Y., Men, X., Xu, Z., Wu, L., Liu, S., et al. 2009. Bile acid transport correlative protein mRNA expression profile in human placenta with intrahepatic cholestasis of pregnancy. Saudi. Med. J. 30 (11): 1406-1410. PMID:19882051.

Ding, L., Yang, L., Wang, Z. and Huang, W. 2015. Bile acid nuclear receptor FXR and digestive system diseases. Acta. Pharm. Sin. B. 5 (2): 135-144. doi: 10.1016/j.apsb.2015.01.004. PMID:26579439.

Donner, M.G., Schumacher, S., Warskulat, U., Heinemann, J. and Häussinger, D. 2007. Obstructive cholestasis induces TNF-alpha- and IL-1 -mediated periportal downregulation of Bsep and zonal regulation of Ntcp, Oatp1a4, and Oatp1b2. Am. J. Physiol. Gastrointest. Liver. Physiol. 293 (6): G1134-1146. doi: 10.1152/ajpgi.00079.2007. PMID: 17916651.

Fu, Z.D., Cui, J.Y. and Klaassen, C.D. 2014. Atorvastatin induces bile acid-synthetic enzyme Cyp7a1 by suppressing FXR signaling in both liver and intestine in mice. J. Lipid. Res. 55 (12): 2576-2586. doi: 10.1194/jlr.M053124. PMID:25278499.

Funk, C., Ponelle, C., Scheuermann, G. and Pantze, M. 2001. Cholestatic potential of troglitazone as a possible factor contributing to troglitazone-induced hepatotoxicity: in vivo and in vitro interaction at the canalicular bile salt export pump (Bsep) in the rat. Mol. Pharmacol. 59 (3): 627-635. PMID:11179459.

Geier, A., Wagner, M., Dietrich, C.G. and Trauner, M. 2007. Principles of hepatic organic anion transporter regulation during cholestasis, inflammation and liver regeneration. Biochim. Biophys. Acta. 1773 (3): 283-308. doi:10.1016/j.bbamcr.2006.04.014. PMID:17291602.

Kajihara, S., Hisatomi, A., Mizuta, T., Hara, T., Ozaki, I., Wada, I., et al. 1998. A splice mutation in the human canalicular multispecific organic anion 
transporter gene causes Dubin-Johnson syndrome. Biochem. Biophys. Res. Commun. 253 (3): 454-457. doi: 10.1006/bbrc.1998.9780. PMID: 9878557.

Kast, H.R., Goodwin, B., Tarr, P.T., Jones, S.A., Anisfeld, A.M., Stoltz, C.M., et al. 2002. Regulation of multidrug resistance-associated protein 2 (ABCC2) by the nuclear receptors pregnane $\mathrm{X}$ receptor, farnesoid X-activated receptor, and constitutive androstane receptor. J. Biol. Chem. 277 (4): 2908-2915. doi: 10.1074/jbc.M109326200. PMID: 11706036.

Knight, J. A. 2005. Liver function tests: their role in the diagnosis of hepatobiliary diseases. J. Infus. Nurs. 28 (2): 108-117. PMID: 15785331.

Kullak-Ublick, G.A., Stieger, B., Hagenbuch, B. and Meier, P.J. 2000. Hepatic transport of bile salts. Semin. Liver. Dis. 20 (3): 273-292. doi: 10.1055/s-2000-9426. PMID: 11076396.

Kwong, E., Li, Y., Hylemon, P.B. and Zhou, H. 2015. Bile acids and sphingosine-1-phosphate receptor 2 in hepatic lipid metabolism. Acta. Pharm. Sin. B. 5 (2): 151-157. doi: 10.1016/j.apsb.2014.12.009. PMID: 26579441.

Lalisang, T. J. 2012. Serum bile acid: an alternative liver function marker in the obstructive jaundice patient. Acta. Med. Indones. 44 (3): 233-238. PMID: 22983079.

Li, G. and Guo, G.L. 2015. Farnesoid X receptor, the bile acid sensing nuclear receptor, in liver regeneration. Acta. Pharm. Sin. B. 5 (2): 93-98. doi: 10.1016/j.apsb.2015.01.005. PMID: 26579433.

Li, W. and Chung, S.C. 2001. An improved rat model of obstructive jaundice and its reversal by internal and external drainage. J. Surg. Res. 101 (1): 4-15. doi: 10.1006/jsre.2001.6240. PMID: 11676548.

Marin, J.J., Macias, R.I., Briz, O., Banales, J. M. and Monte, M. J. 2015. Bile Acids in Physiology, Pathology and Pharmacology. Curr. Drug. Metab. 17 (1): 4-29. doi: 10.1006/jsre.2001.6240. PMID: 11676548.

Matsuzaka, Y., Hayashi, H. and Kusuhara, H. 2015. Impaired Hepatic Uptake by Organic Anion-Transporting Polypeptides Is Associated with Hyperbilirubinemia and Hypercholanemia in Atp11c Mutant Mice. Mol. Pharmacol. 88 (6): 1085-1092. doi: 10.1124/mol.115.100578. PMID: 26399598.

Mazuy, C., Helleboid, A., Staels, B. and Lefebvre, P. 2015. Nuclear bile acid signaling through the farnesoid X receptor. Cell. Mol. Life. Sci. 72 (9): 1631-1650. doi: 10.1007/s00018-014-1805-y. PMID: 25511198.

Miller, A. L. 1999. Therapeutic considerations of L-glutamine: a review of the literature. Altern. Med. Rev. 4 (4): 239-248. PMID: 10468648.

Morgan, A.E., Mooney, K.M., Wilkinson, S.J., Pickles, N.A. and Mc Auley, M.T. 2016. Cholesterol metabolism: A review of how ageing disrupts the biological mechanisms responsible for its regulation. Ageing. Res. Rev. 27 (1): 108-124. doi: 10.1016/j.arr.2016.03.008. PMID: 27045039.

Sang, Y., Yang, J., Ross, C.R., Rowland, R.R. and Blecha, F. 2008. Molecular 
463

464

465

466

467

468

469

470

471

472

473

474

475

476

477

478

479

480

481

482

483

484

485

486

487

488

489

490

491

492

493

494

495

496

497

498

499

500

501

502

503

504

identification and functional expression of porcine Toll-like receptor (TLR) 3 and TLR7. Vet. Immunol. Immunopathol. 125 (1-2): 162-167. doi: 10.1016/j.vetimm.2008.04.017. PMID: 18533275.

Scheig, R. 1996. Evaluation of tests used to screen patients with liver disorders. Prim. Care. 23 (3): 551-560. PMID: 8888344.

Shen, L., Hillebrand, A., Wang, D.Q. and Liu, M. 2012. Isolation and primary culture of rat hepatic cells. J. Vis. Exp. 29 (64): pii: 3917. doi: 10.3791/3917. PMID: 22781923.

Song, X., Chen, Y., Valanejad, L., Kaimal, R., Yan, B., Stoner, M., et al. 2013. Mechanistic insights into isoform-dependent and species-specific regulation of bile salt export pump by farnesoid X receptor. J. Lipid. Res. 54 (11): 3030-3044. doi: 10.1194/jlr.M038323. PMID: 24002920.

Sorrentino, P., Tarantino, G., Perrella, A., Micheli, P., Perrella, O. and Conca, P. 2005. A Clinical-Morphological Study on Cholestatic Presentation of Nonalcoholic Fatty Liver Disease. Dig. Dis. Sci. 50 (6): 1130-1135. doi:10.1007/s10620-005-2719-1. PMID: 15986869.

Stieger, B. 2011. The role of the sodium-taurocholate cotransporting polypeptide (NTCP) and of the bile salt export pump (BSEP) in physiology and pathophysiology of bile formation. Handb. Exp. Pharmacol. 201 (1): 205-259. doi: 10.1007/978-3-642-14541-4_5. PMID: 21103971.

Stieger, B. and Beuers, U. 2011. The canalicular bile salt export pump BSEP (ABCB11) as a potential therapeutic target. Curr. Drug Targets 12 (5): 661-670. PMID: 21039337.

Suchy, F.J. and Ananthanarayanan, M. 2006. Bile salt excretory pump: biology and pathobiology. J. Pediatr. Gastroenterol. Nutr. 43 (suppl 1): S10-16. doi: 10.1097/01.mpg.0000226385.71859.5f. PMID: 16819395.

Tarantino, G., Conca, P., Basile, V., Gentile, A., Capone, D., Polichetti, G., et al. 2007. A prospective study of acute drug-induced liver injury in patients suffering from non-alcoholic fatty liver disease. Hepatol. Res. 37 (6): 410-415. doi:10.1111/j.1872-034X.2007.00072.x. PMID: 17539815.

Trauner, M., Meier, P.J. and Boyer, J.L. 1998. Molecular pathogenesis of cholestasis. N. Engl. J. Med. 339 (17): 1217-1227. doi: 10.1056/NEJM199810223391707. PMID: 9780343.

Trauner, M.M., Wagner, M.M., Fickert, P.M. and Zollner, G.M. 2005. Molecular Regulation of Hepatobiliary Transport Systems: Clinical Implications for Understanding and Treating Cholestasis. J. Clin. Gastroenterol. 39 (4): S111-S124. PMID: 15758646.

Wendum, D. 2010. [Liver disease associated with hereditary defects of hepatobiliary transporters]. Ann. Pathol. 30 (6): 426-431. doi: 10.1016/j.annpat.2010.08.025. PMID: 21167428.

Xu, F., Dai, C.L., Peng, S.L., Zhao, Y., Jia, C.J. and Xu, Y.Q. 2014. Preconditioning with Glutamine Protects against Ischemia/Reperfusion-Induced Hepatic Injury 
505

506

507

508

509

510

511

512

513

514

515

516

517

518

519

520

521

522

523

524

525

526

527

528

529

530

531

in Rats with Obstructive Jaundice. Pharmacology, 93 (3-4): 155-165. doi:10.1159/000360181. PMID: 24801881.

Zhou, H. and Hylemon, P.B. 2014. Bile acids are nutrient signaling hormones. Steroids, 86 (1): 62-68. 10.1016/j.steroids.2014.04.016. PMID: 24819989.

Zollner, G., Marschall, H.U., Wagner, M. and Trauner, M. 2006. Role of nuclear receptors in the adaptive response to bile acids and cholestasis: pathogenetic and therapeutic considerations. Mol. Pharm. 3 (3): 231-251. doi: 10.1021/mp060010s. PMID: 16749856.

Zollner, G. and Trauner, M. 2006. Molecular mechanisms of cholestasis. Wien. Med. Wochenschr. 156 (13-14): 380-385. doi:10.1007/s10354-006-0312-7. PMID: 16937039 .

.


Table 1 PCR primer sequences and the length of the amplified products

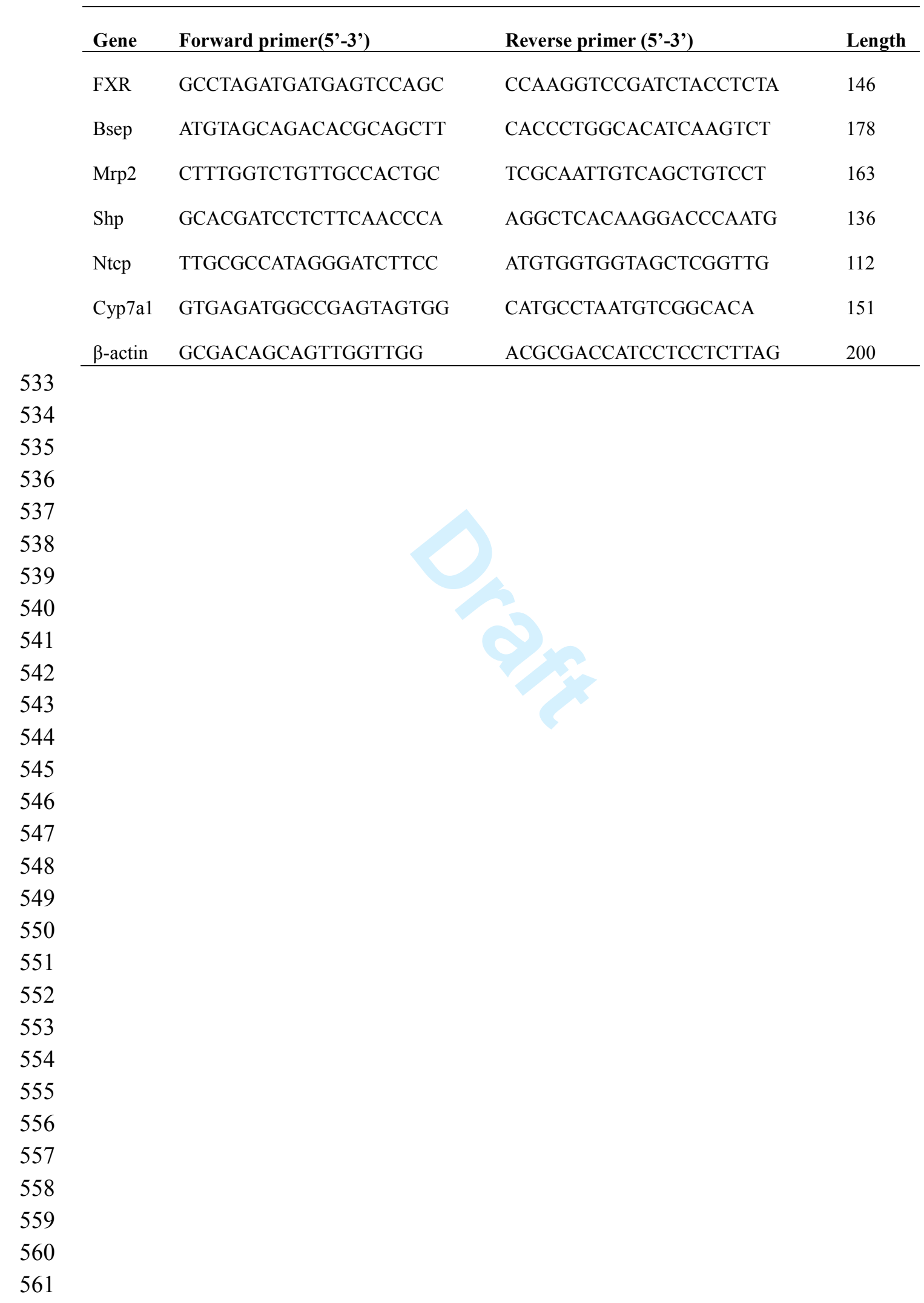


Table 2 The results of liver histopathological examination grade

\begin{tabular}{lllll}
\hline Group & $\begin{array}{l}\text { Inflammatory cell } \\
\text { infiltration }\end{array}$ & $\begin{array}{l}\text { Hepatocyte } \\
\text { necrosis }\end{array}$ & $\begin{array}{l}\text { Bile duct } \\
\text { proliferation }\end{array}$ & $\begin{array}{l}\text { Liver } \\
\text { fibrosis }\end{array}$ \\
\hline SH+NS & - & - & - & - \\
BDL+NS & +++ & ++ & +++ & +++ \\
BDL+Gln & + & - & + & - \\
BDL+UDCA & + & - & + & - \\
\hline
\end{tabular}

563

564

565

566

567

568

569

570

571

572

573

574

575

576

577

578

579 
581 Fig. 1. Detection of biochemical indicator levels in four rat groups with different treatments.

582 Serum ALT (A), AST (B), ALP (C), $\gamma$-GTP (D) activity as well as serum TC (E), DBIL (F),

583 TBIL (G) and TBA (H) levels were detected with automatic biochemical analyzer. Data are

584 the mean $\pm \mathrm{SD} . * * P<0.01$ vs. $\mathrm{SH}+\mathrm{NS} ;{ }{ }^{\#} P<0.01$ vs. BDL+NS.

585 Fig. 2. The images of representative H\&E stained liver sections (200×magnification) were

586 shown from $\mathrm{SH}+\mathrm{NS}, \mathrm{BDL}+\mathrm{NS}, \mathrm{BDL}+\mathrm{Gln}$, and BDL+UDCA groups.

587 Fig. 3. Immunohistochemical staining of Bsep and Mrp2 protein distributions in liver tissues

588 of BDL rats with Gln treatment (400×magnification; bar $=50 \mu \mathrm{m})$.(A) Representative images

589 of immunohistochemical staining for Bsep and Mrp2. (B) IOD values of Bsep and Mrp2 in

590 rats liver tissue. The IOD values were examed were analyzed by microscope in six random

591 fields. Data are the mean $\pm \mathrm{SD} .{ }^{* *} P<0.01$ vs. $\mathrm{SH}+\mathrm{NS}$; ${ }^{\# \#} P<0.01$ vs. BDL+NS.

592 Fig. 4. Alternation of BAs regulatory gene expression changes in BDL rats with Gln

593 treatment. (A) Protein levels of FXR, Bsep, Mrp2, Shp, Ntcp, and Cyp7a1 change detected

594 was by western blot. (B) Relative protein expression levels detected in (A). (C) mRNA levels

595 of FXR, Bsep, Mrp2, Shp, Ntcp, and Cyp7a1change was detected by RT-PCR. Data are the

596 mean $\pm \mathrm{SD} .{ }^{* *} P<0.01$ vs. $\mathrm{SH}+\mathrm{NS} ;{ }^{\# \#} P<0.01$ vs. BDL+NS.

597 Fig. 5. In vitro evidence on FXR activation by Gln. (A) FXR knockdown abrogated the

598 regulation of Bsep and Mrp2 by Gln in rat primary hepatocytes. (B) Relative protein

599 expression levels detected in (A). (C) Luciferase reporter assay performed in HepG2 cells

600 with FXR expression vector and Bsep promoter reporter. Data are the mean \pm SD. ${ }^{*} P<0.05$, 
$601 \quad * * P<0.01$ vs. vehicle; ${ }^{\#} P<0.01$ vs. Gln treatment alone. 

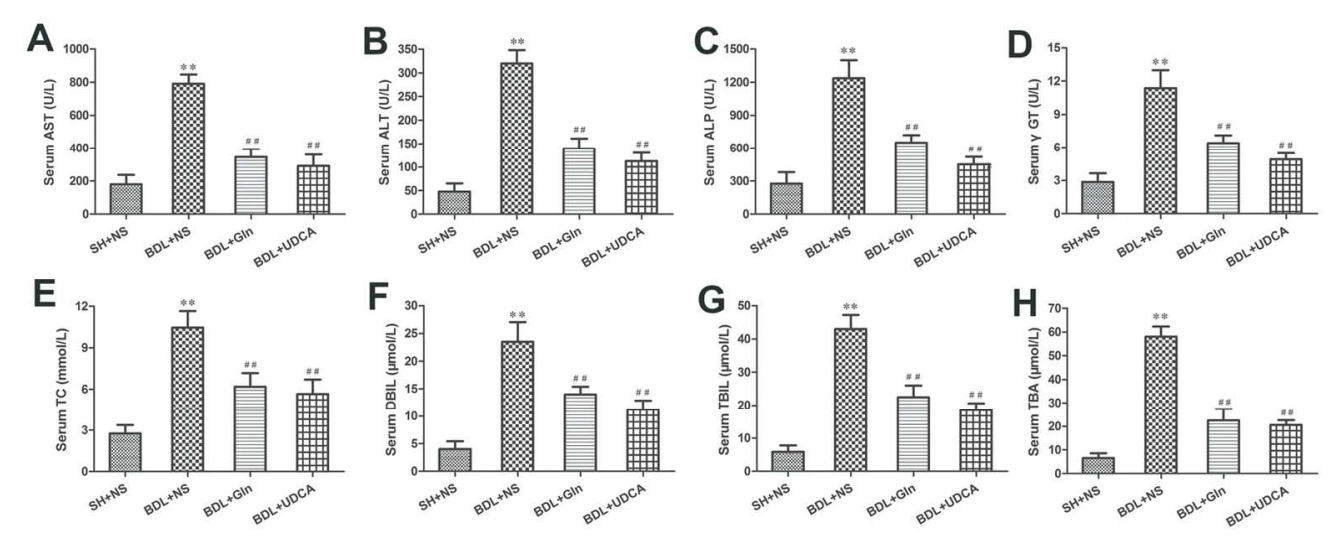

Fig1.TIF

$67 \times 27 \mathrm{~mm}(600 \times 600 \mathrm{DPI})$ 

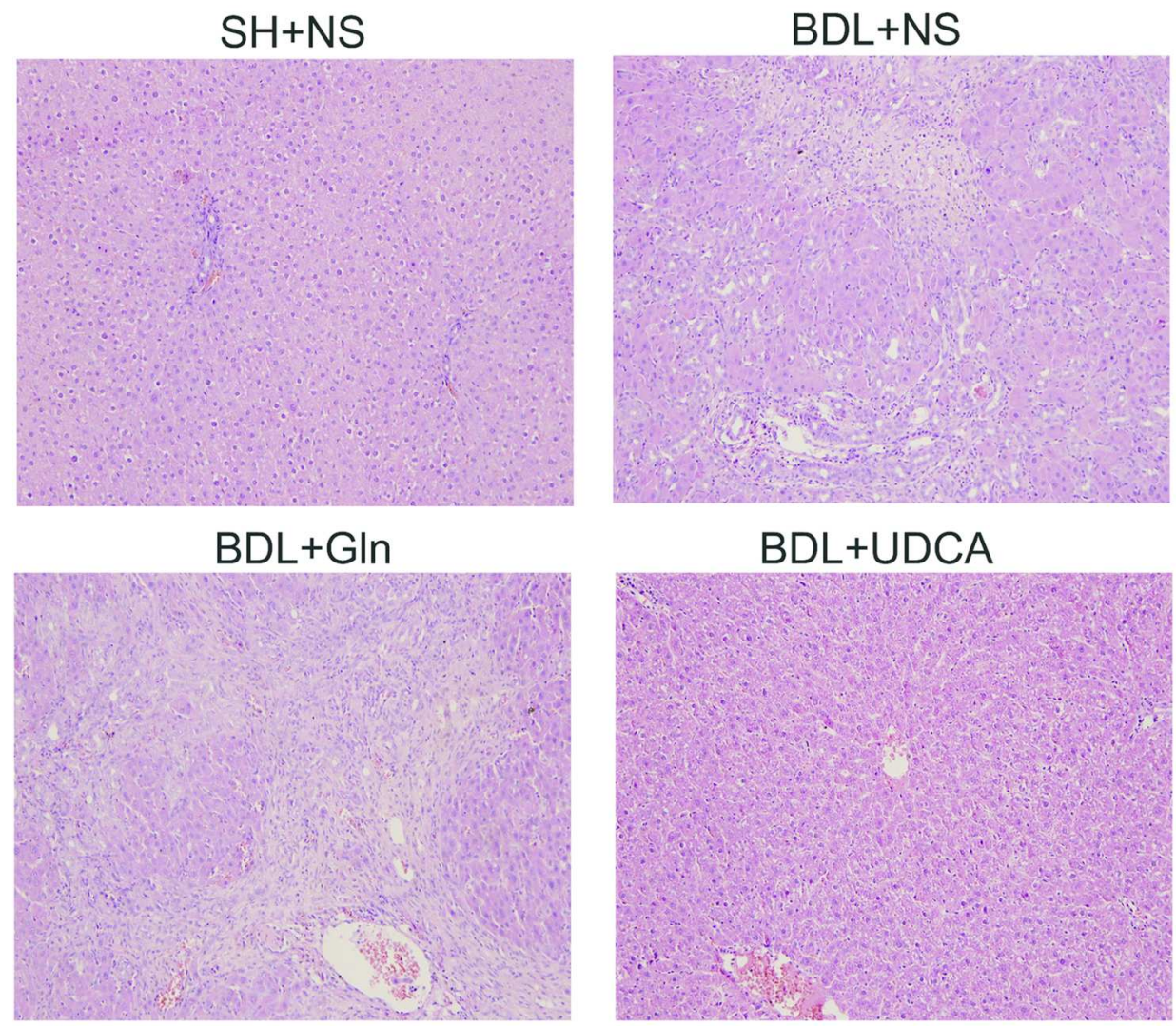

Fig2. TIF

$68 \times 59 \mathrm{~mm}(600 \times 600 \mathrm{DPI})$ 

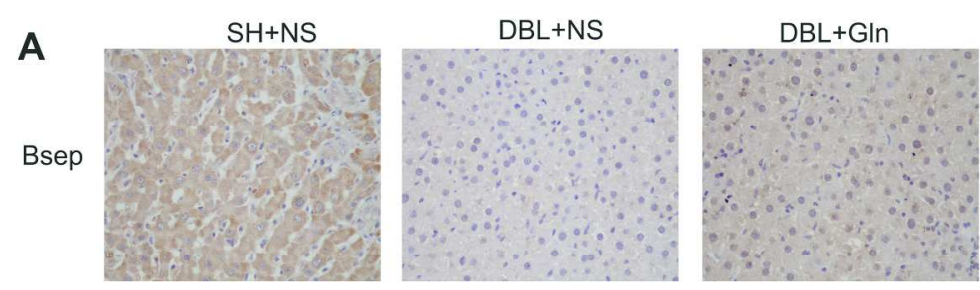

$D B L+U D C A$
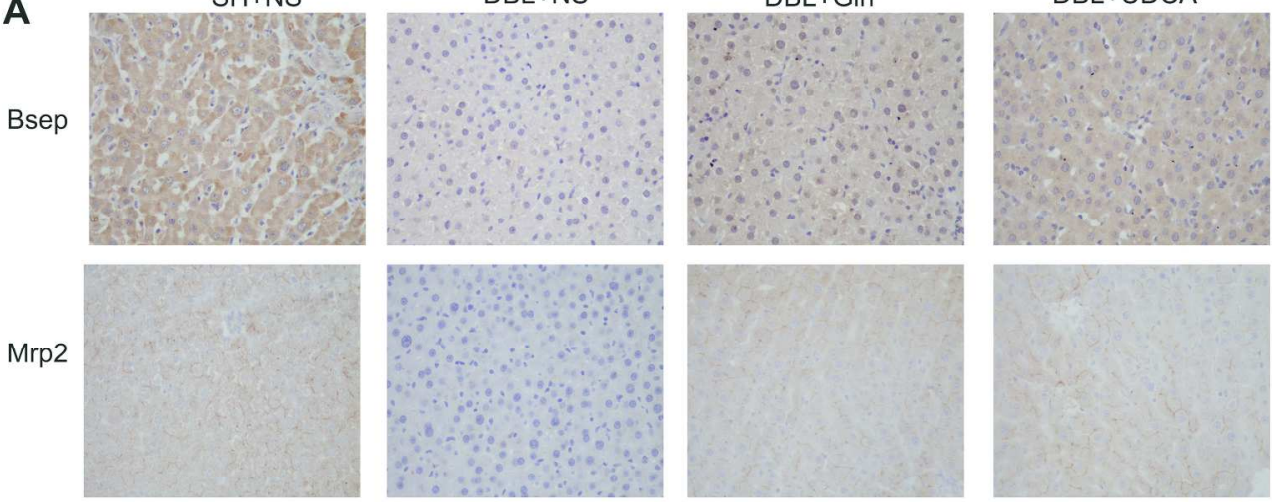

в
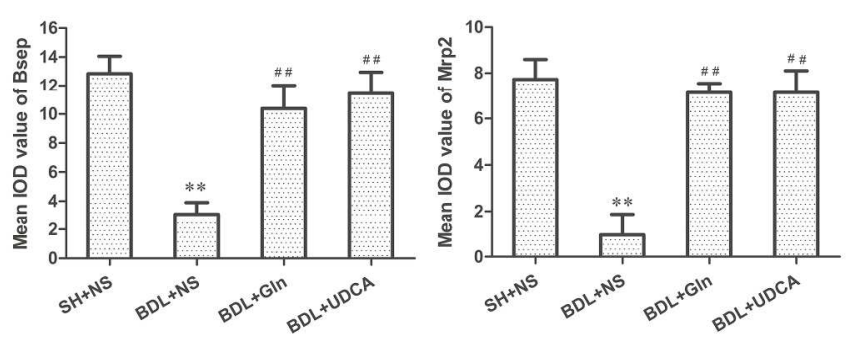

Fig3. TIF

$117 \times 81 \mathrm{~mm}(600 \times 600 \mathrm{DPI})$ 


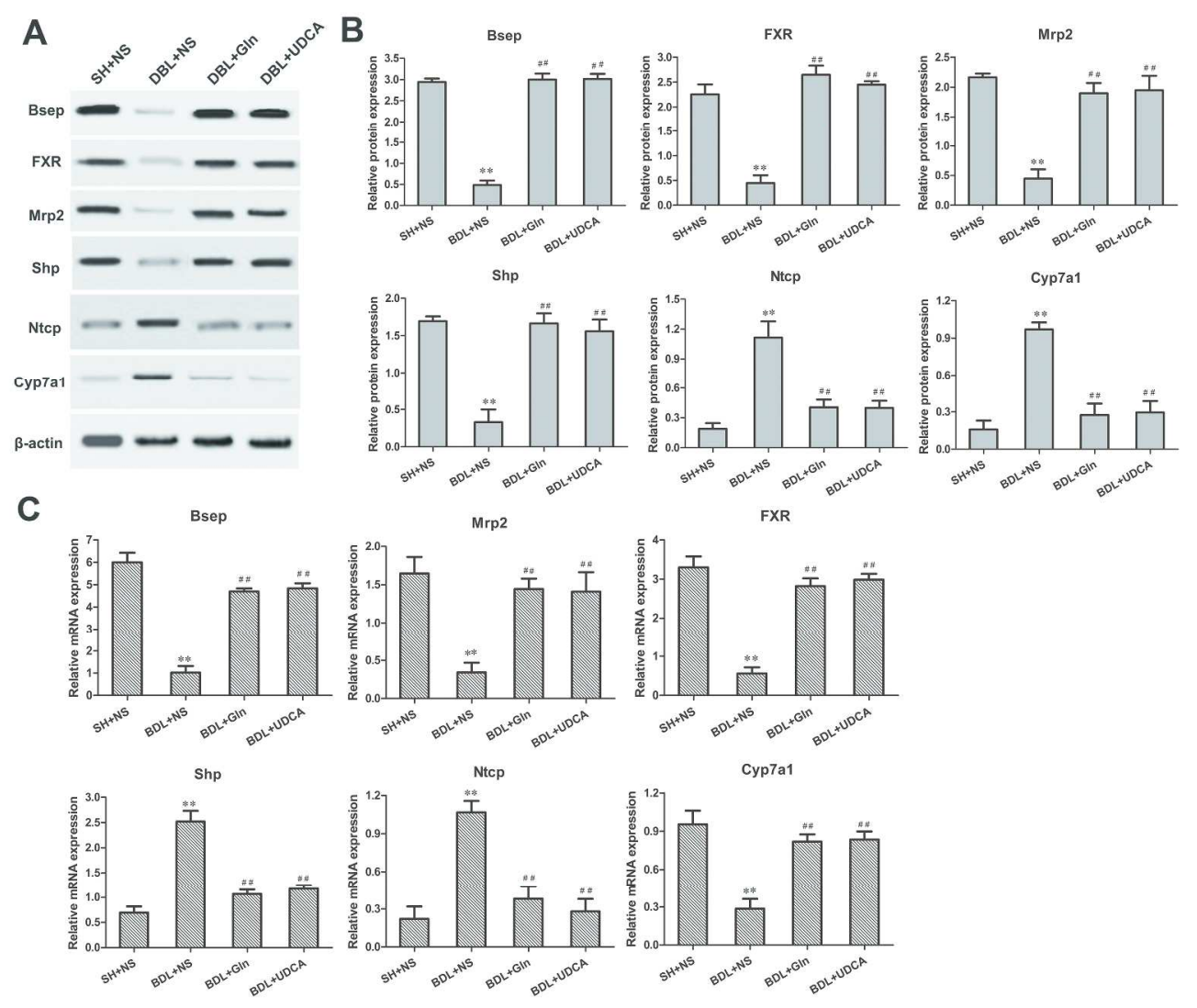

Fig4.TIF

$143 \times 121 \mathrm{~mm}(600 \times 600 \mathrm{DPI})$ 


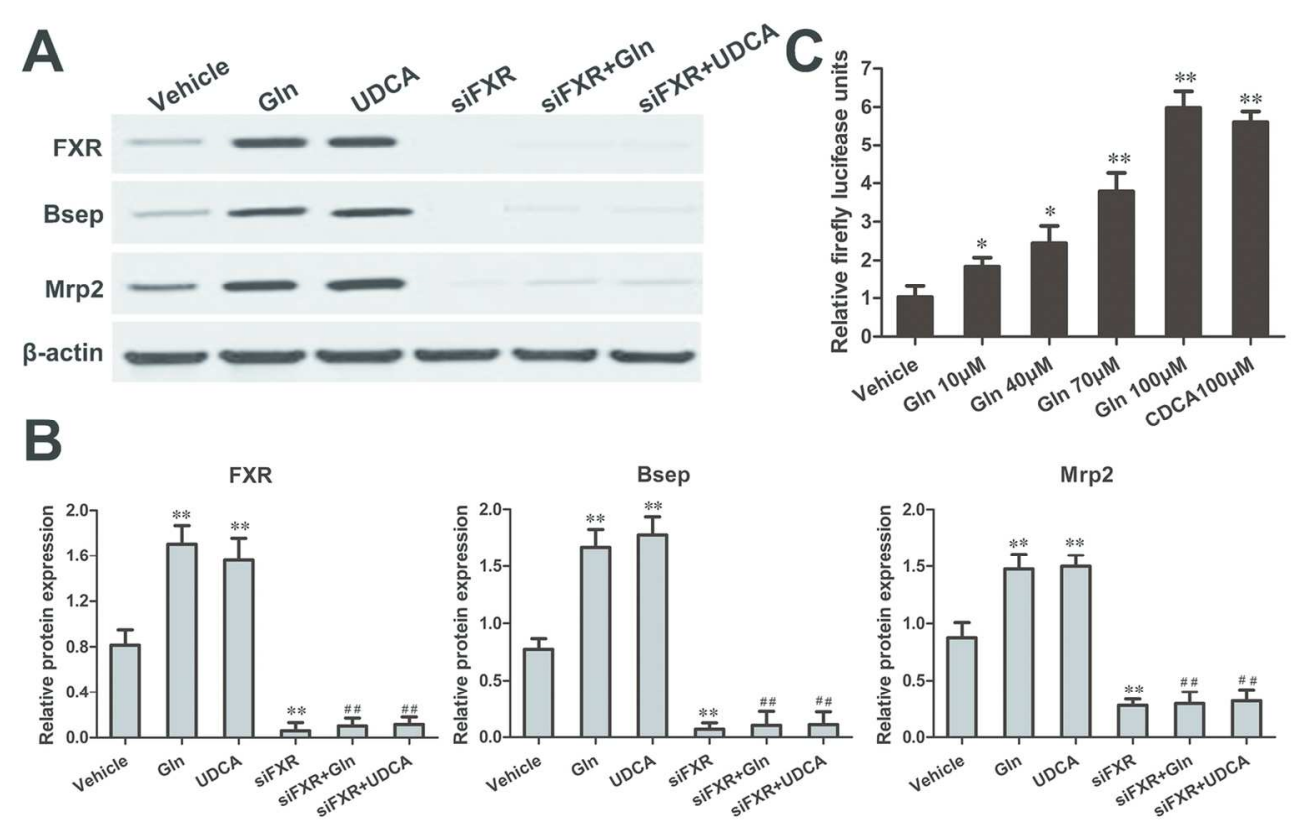

Fig5.TIF

$68 \times 42 \mathrm{~mm}(600 \times 600 \mathrm{DPI})$ 